\title{
Synthesis and Antiproliferative Activity of Steroidal Thiosemicarbazone Platinum (Pt(II)) Complexes
}

\author{
Yanmin Huang, ${ }^{1}$ Erbin Kong, ${ }^{1}$ Chunfang Gan, ${ }^{1}$ Zhiping Liu, ${ }^{1}$ Qifu Lin, ${ }^{2}$ and Jianguo Cui ${ }^{1,2}$ \\ ${ }^{1}$ College of Chemistry and Materials Science, Guangxi Teachers Education University, Nanning 530001, China \\ ${ }^{2}$ Guangxi Colleges and University Key Laboratory of Beibu Gulf Oil and Natural Gas Resource Effective Utilization, \\ Qizhou University, Qizhou 535000, China
}

Correspondence should be addressed to Jianguo Cui; cuijg1954@126.com

Received 21 July 2015; Accepted 17 September 2015

Academic Editor: Giovanni Natile

Copyright (C) 2015 Yanmin Huang et al. This is an open access article distributed under the Creative Commons Attribution License, which permits unrestricted use, distribution, and reproduction in any medium, provided the original work is properly cited.

\begin{abstract}
Steroidal compounds exhibit particular physiological activities. In this paper, some steroidal thiosemicarbazones platinum (Pt(II)) complexes were synthesized by the condensation of steroidal ketones with thiosemicarbazide using estrone, chenodeoxycholic acid, and 7-deoxycholic acid as starting materials and complexation of steroidal thiosesemicarbazones with Pt(II). The complexes were characterized by IR, NMR, and MS, and their antiproliferative activities were evaluated. The results showed that some steroidal thiosemicarbazones platinum ( $\mathrm{Pt}(\mathrm{II})$ ) complexes displayed moderate cytotoxicity to HeLa and Bel-7404 cells. Thereinto, complex 6 showed an excellent inhibited selectivity to HeLa cells with an $\mathrm{IC}_{50}$ value of $9.2 \mu \mathrm{M}$ and SI value of 21.7. At the same time, all compounds were almost inactive to HEK293T (normal kidney epithelial cells). The information obtained from the studies may be useful for the design of novel chemotherapeutic drugs.
\end{abstract}

\section{Introduction}

In the late 1960s, anticancer activity of cisplatin was found by Rosenberg [1-4]. Subsequently, cisplatin had become a metal anticancer drug for the treatment of human cancers. But in the process of treatment, cisplatin showed a high toxicity to patients and led to some strong side effects [5]. In accordance with the traditional structure-activity relationship of platinum complexes, the synthesis of new platinum anticancer drugs with the same mode of action has difficulty in achieving a major breakthrough. Because steroid hormones play an important role in the biochemistry of many cancers, a number of steroidal complexes connected to a metal pharmacophore had been designed and synthesized by many research groups, and their physiological activities were evaluated [615]. Steroidal metal complexes are transferred to cancer cells by steroidal carrier, selectively accumulated in cancer cells, and combined with specific DNA to reduce the destruction of normal cells. Not only can the metal pharmacophores of steroidal metal complexes enhance DNA damage by its space hinder, but also they play the role of steroid hormones and interfere with cancer cell growth regulating process. Therefore, maintaining and improving the two parts of the activity of the steroidal part and platinum pharmacophore are the key to the molecular design strategy of anticancer steroidal platinum complexes [16-18]. Thiosemicarbazones have received considerable attention since the discovery of their cytotoxic activity against cancer cells and bacteriostatic effects, and the biological properties of thiosemicarbazone complexes are often related and modulated by the metal ion of coordination [19]. Khan and Yusuf [20] and Murugkar et al. [21] investigated the bioactivity of some new steroidal thiosemicarbazones and their $\mathrm{Pd}(\mathrm{II})$ or $\mathrm{Pt}(\mathrm{II})$ metal complexes and discovered that some compounds had better antibacterial or antineoplastic activity.

In the present study, some novel steroidal thiosemicarbazone platinum $(\mathrm{Pt}(\mathrm{II}))$ complexes were synthesized by condensing steroidal ketones with thiosemicarbazide using estrone, chenodeoxycholic acid, and 7-deoxycholic acid as starting materials and complexing steroidal thiosemicarbazone with $\mathrm{Pt}(\mathrm{II})$. Their antiproliferative activities against HeLa (human cervical carcinoma), Bel-7404 (human liver 
carcinoma), and HEK293T (normal kidney epithelial cells) cells were evaluated.

\section{Materials and Methods}

2.1. Materials. The sterols were purchased from Sinopharm Chemical Reagent Co., Ltd., Shanghai, China. All chemicals and solvents were of analytical grade from commercial sources. All solvents were used without further purification unless otherwise specified.

2.2. Instrumentation and Methods. Melting points were determined on an $\mathrm{X}_{4}$ apparatus (Beijing Tech Instrument Co. Ltd., Beijing, China) and were uncorrected. The ${ }^{1} \mathrm{H}$ and ${ }^{13} \mathrm{C}$ NMR spectra were recorded in $\mathrm{CDCl}_{3}$ on a Bruker AV-600 spectrometer at working frequencies 600 and $150 \mathrm{MHz}$ and a Bruker AV-300 spectrometer at working frequencies 300 and $75 \mathrm{MHz}$, respectively. Chemical shifts are expressed in parts per million $(\delta)$ values and coupling constants $(J)$ in Hertz. Infrared spectra were measured with a Thermo Scientific Nicolet IS-10 Spectrophotometer (Thermo Scientific, America). HREIMS was measured on an Agilent 6210 TOFMS instrument (Agilent Technologies, America). The cell proliferation assay was undertaken by a MTT method using 96well plates on a MLLTISKAN MK3 analysis spectrometer (Thermo Scientific, Shanghai, China).

2.3. Synthesis. Compound 7 was prepared according to the method of [22] and compound $\mathbf{1 2}$ was prepared following the method of [23].

2.3.1. General Procedure for Preparation of Steroidal Thiosemicarbazone. A mixture of steroidal ketone $(1 \mathrm{mmol})$, thiosemicarbazide $(1 \mathrm{mmol})$, and a few drops of glacial acetic acid in $95 \%$ ethanol $(30 \mathrm{~mL})$ was stirred at $60-70^{\circ} \mathrm{C}$ for $10 \mathrm{~h}$. After completion of the reaction, the majority of solvent was evaporated and some water was added to this solution. The mixture was extracted with $\mathrm{CH}_{2} \mathrm{Cl}_{2}$ and the extract was washed with saturated brine, dried with anhydrous sodium sulfate, and evaporated under reduced pressure. The resulting residue was chromatographed on a column of silica gel with a mixture of DCM : methanol $(20: 1)$ to give steroidal thiosemicarbazone.

3ß-Hydroxyestron-17-thiosemicarbazone (2, $\left.L^{2}\right)$. White solid, Yield: $78.4 \%$; m.p. $254-256^{\circ} \mathrm{C}$; IR (KBr) $v / \mathrm{cm}^{-1}$ : 3419, 3359, $3159,2927,1718,1574,1464,1434 ;{ }^{1} \mathrm{H}$ NMR $(600 \mathrm{MHz}$, DMSO) $\delta: 0.827\left(3 \mathrm{H}, \mathrm{s}, 18-\mathrm{CH}_{3}\right), 2.567(1 \mathrm{H}, \mathrm{dd}, J=19.2$, 9.0, C6-H), 2.77-2.67 (2H, m, C16-H), $6.436(1 \mathrm{H}, \mathrm{d}, J=2.4$, C4-H), $6.505(1 \mathrm{H}, \mathrm{dd}, J=8.4,2.4, \mathrm{C} 2-\mathrm{H}), 7.044(1 \mathrm{H}, \mathrm{d}, J=8.4$, $\mathrm{Cl}-\mathrm{H}), 7.397\left(1 \mathrm{H}, \mathrm{s},-\mathrm{NH}_{2}\right), 8.046\left(1 \mathrm{H}, \mathrm{s},-\mathrm{NH}_{2}\right), 9.030(1 \mathrm{H}$, s, -NH-), $9.880(1 \mathrm{H}, \mathrm{s},-\mathrm{OH}) ;{ }^{13} \mathrm{C}$ NMR (150 MHz, DMSO) $\delta$ : 17.0 (18-C), 22.9 (11-C), 26.0 (15-C), 26.9 (16-C), 27.0 (7-C), 29.2 (6-C), 33.9 (12-C), 38.0 (9-C), 43.7 (8-C), 44.8 (13-C), 51.9 (14-C), 112.9 (2-C), 115.1 (4-C), 126.2 (1-C), 130.2 (10-C), 137.2 (5-C), 155.1 (3-C), 167.4 (17-C), 178.4 (C=S); HREIMS: $m / z 343.1718[\mathrm{M}+\mathrm{H}]^{+}\left(\right.$calcd for $\left.\mathrm{C}_{19} \mathrm{H}_{25} \mathrm{~N}_{3} \mathrm{OS}, 343.1718\right)$.

$3 \beta$-Acetyloxyestron-17-thiosemicarbazone $\left(5, L^{5}\right)$. White solid, Yield: $77.8 \%$; m.p. $248-249^{\circ} \mathrm{C}$; IR $(\mathrm{KBr}) \nu / \mathrm{cm}^{-1}$ : 3237, 2930,
$1766,1584,1516,1484,1369,1147,936,813 ;{ }^{1} \mathrm{H}$ NMR $(600 \mathrm{MHz}$, $\left.\mathrm{CDCl}_{3}\right) \delta: 0.907\left(3 \mathrm{H}, \mathrm{s}, 18-\mathrm{CH}_{3}\right), 2.277\left(3 \mathrm{H}, \mathrm{s},-\mathrm{OCH}_{3}\right), 2.40-$ $2.37(1 \mathrm{H}, \mathrm{m}, \mathrm{C} 6-\mathrm{H}), 2.481(1 \mathrm{H}, \mathrm{dd}, J=18.0,8.4, \mathrm{C} 6-\mathrm{H}), 2.90-$ $2.87(2 \mathrm{H}, \mathrm{m}, \mathrm{Cl}-\mathrm{H}), 6.435\left(1 \mathrm{H}, \mathrm{s},-\mathrm{NH}_{2}\right), 6.798(1 \mathrm{H}, \mathrm{d}, J=$ 2.4, C4-H), $6.845(1 \mathrm{H}, \mathrm{dd}, J=8.4,2.4, \mathrm{C} 2-\mathrm{H}), 7.205(1 \mathrm{H}, \mathrm{s}$, $\left.-\mathrm{NH}_{2}\right), 7.276(1 \mathrm{H}, \mathrm{d}, J=8.4, \mathrm{C} 1-\mathrm{H}), 8.437(1 \mathrm{H}, \mathrm{s},-\mathrm{NH}-) ;{ }^{13} \mathrm{C}$ NMR (75 MHz, DMSO) $\delta$ : 17.1 (18-C), $21.2\left(\mathrm{CH}_{3} \mathrm{CO}\right), 23.3(11-$ C), 26.0 (15-C), 26.4 (16-C), 27.0 (7-C), 29.4 (6-C), 34.0 (12C), 37.8 (8-C), 44.2 (9-C), 45.1 (13-C), 52.5 (14-C), 118.8 (2-C), 121.7 (4-C), 126.4 (1-C), 137.5 (5-C), 138.1 (10-C), 148.6 (3-C), $167.3(17-\mathrm{C}), 170.0\left(\mathrm{COCH}_{3}\right), 178.9(\mathrm{C}=\mathrm{S})$; HREIMS: $[\mathrm{M}+\mathrm{H}]^{+}$ 408.1736 (calcd for $\mathrm{C}_{21} \mathrm{H}_{27} \mathrm{~N}_{3} \mathrm{NaO}_{2} \mathrm{~S}, 408.1772$ ).

Methyl 3-Thiosemicarbazonyl-7-oxochenodeoxycholicate (8, $\left.L^{8}\right)$. Light yellow solid, Yield: $53.0 \%, m . p .285-286^{\circ} \mathrm{C}$; IR (KBr) $v / \mathrm{cm}^{-1}: 2947,2868,1706,1624,1434,1329,1170,1023 ;{ }^{1} \mathrm{H}$ $\mathrm{NMR}\left(\mathrm{CDCl}_{3}, 600 \mathrm{MHz}\right): 0.65\left(3 \mathrm{H}, \mathrm{s}, 18-\mathrm{CH}_{3}\right), 0.89(1.2 \mathrm{H}, \mathrm{d}$, $\left.J=6.6,21-\mathrm{CH}_{3}, 3 \mathrm{Z}\right), 0.90\left(1.8 \mathrm{H}, \mathrm{d}, J=6.6,21-\mathrm{CH}_{3}, 3 E\right), 1.22$ $\left(1.8 \mathrm{H}, \mathrm{s}, 19-\mathrm{CH}_{3}, 3 \mathrm{E}\right), 1.23\left(1.2 \mathrm{H}, \mathrm{s}, 19-\mathrm{CH}_{3}, 3 \mathrm{Z}\right), 2.31(0.6 \mathrm{H}$, $\mathrm{dd}, J=10.2,5.4, \mathrm{C} 4-\beta \mathrm{H}, 3 E), 2.34(0.4 \mathrm{H}, \mathrm{dd}, J=10.2,5.4, \mathrm{C} 4-$ $\beta \mathrm{H}, 3 \mathrm{Z}), 2.84(0.6 \mathrm{H}, \mathrm{dd}, J=13.2,6.0, \mathrm{C} 6-\beta \mathrm{H}, 3 \mathrm{E}), 2.89(0.4 \mathrm{H}$, dd, $J=13.2,6.0, \mathrm{C} 6-\beta \mathrm{H}, 3 \mathrm{Z}), 3.63\left(3 \mathrm{H}, \mathrm{s}, \mathrm{OCH}_{3}\right), 6.52(1 \mathrm{H}$, br s, $\left.-\mathrm{NH}_{2}\right), 7.19\left(0.4 \mathrm{H}, \mathrm{d}, J=4.2,-\mathrm{NH}_{2}, 3 Z\right), 7.19(0.6 \mathrm{H}, \mathrm{d}$, $\left.J=4.2,-\mathrm{NH}_{2}, 3 E\right), 8.93(0.6 \mathrm{H}, \mathrm{s},-\mathrm{NH}-, 3 E), 8.97(0.4 \mathrm{H}, \mathrm{s}$, $\mathrm{NH}-, 3 Z) ;{ }^{13} \mathrm{C}$ NMR $\left(\mathrm{CDCl}_{3}, 150 \mathrm{MHz}\right): 211.7$ (7-C, 3E), 211.5 (7-C, 3Z), 178.9 (C=S), 174.8 (24-C), 155.4 (3-C, 3E), 155.3 (3C, 3Z), 54.9 (17-C), $51.7\left(\mathrm{O}-\mathrm{CH}_{3}\right), 49.6$ (9-C), 49.0 (8-C, 3E), 48.9 (8-C, 3Z), 47.7 (14-C), 46.8 (5-C, 3Z), 45.0 (5-C, 3E), 43.1 (13-C), 42.8 (10-C), 42.7 (6-C), 38.9 (12-C), 36.5 (20-C), 35.9 (4-C, 3-E), 35.3 (4-C, 3-Z), 31.2 (22-C), 31.1 (23-C), 29.8 (2-C, 3E), 28.7 (2-C, 3Z), 28.4 (16-C), 24.9 (1-C, 3E), 24.8 (1-C, 3Z), 22.9 (15-C), 22.1 (11-C), 18.5 (21-C), 14.3 (19-C), 12.2 (18-C); HREIMS: $m / z$ 476.2941 $[\mathrm{M}+\mathrm{H}]^{+}\left(\right.$calcd for $\mathrm{C}_{26} \mathrm{H}_{42} \mathrm{~N}_{2} \mathrm{O}_{3} \mathrm{~S}$, 476.2947).

Methyl 3-Thiosemicarbazonyl-12-oxo-7-deoxycholicate (13, $\left.L^{13}\right)$. Light yellow solid, Yield: $52.5 \%$, m.p. $125-127^{\circ} \mathrm{C}$; IR $(\mathrm{KBr}) v / \mathrm{cm}^{-1}: 3431,1736,1706,1591,1494 ;{ }^{1} \mathrm{H}$ NMR $\left(\mathrm{CDCl}_{3}\right.$, $300 \mathrm{MHz}): 0.841\left(3 \mathrm{H}, \mathrm{d}, J=6.3,21-\mathrm{CH}_{3}\right), 1.034(3 \mathrm{H}, \mathrm{s}$, $\left.19-\mathrm{CH}_{3}\right), 1.062\left(3 \mathrm{H}, \mathrm{s}, 18-\mathrm{CH}_{3}\right), 2.60-2.49(2 \mathrm{H}, \mathrm{m}, \mathrm{Cl1}-\mathrm{H})$, $3.657\left(3 \mathrm{H}, \mathrm{s}, \mathrm{OCH}_{3}\right), 6.496\left(1 \mathrm{H}, \mathrm{br} \mathrm{s},-\mathrm{NH}_{2}\right), 7.214(1 \mathrm{H}, \mathrm{br} \mathrm{s}$, $\left.-\mathrm{NH}_{2}\right), 8.897(1 \mathrm{H}, \mathrm{s},-\mathrm{NH}) ;{ }^{13} \mathrm{C}$ NMR $\left(\mathrm{CDCl}_{3}, 150 \mathrm{MHz}\right)$ : 11.7 (18-C), 18.6 (19-C), 22.3 (21-C), 24.3 (15-C), 25.6 (16-C), 27.5 (1-C), 29.8 (2-C), 30.5 (6-C), 31.3 (7-C), 35.4 (22-C), 35.6 (23-C), 36.0 (4-C), 37.0 (20-C), 38.3 (11-C), 42.3 (8-C), 43.7 (10-C), 44.4 (13-C), 46.5 (5-C), $51.5\left(\mathrm{OCH}_{3}\right), 57.5$ (9-C), 58.4 (17-C), 58.5 (14-C), 156.9 (3-C), 174.6 (24-C), $178.7(\mathrm{C}=\mathrm{S})$, 214.3 (12-C); HREIMS: $m / z$ 476.2942 $[\mathrm{M}+\mathrm{H}]^{+}$(calcd for $\left.\mathrm{C}_{26} \mathrm{H}_{42} \mathrm{~N}_{3} \mathrm{O}_{3} \mathrm{~S}, 476.2947\right)$.

2.3.2. Methyl 3-Thiosemicarbazonyl-7-hydroxychenodeoxycholicate $\left(10, L^{10}\right)$. To the stirred solution of $\mathbf{8}(674 \mathrm{mg}$, $1.42 \mathrm{mmol}$ ) in $\mathrm{CH}_{3} \mathrm{OH}(30 \mathrm{~mL})$ was added $\mathrm{NaBH}_{4}$ (96 mg, $2.52 \mathrm{mmol}$ ) in $10 \mathrm{~min}$ at room temperature. After no starting material was observed (the progress of the reaction was monitored by TLC, petroleum ether/ethyl acetate $=1: 1$ ), the reaction was stopped. The solution was neutralized with $1 \mathrm{M} \mathrm{HCl}$. After evaporation of the majority of $\mathrm{MeOH}$ under reduced pressure, proper water was added. The residue 
was extracted with ethyl acetate. The organic layer was washed with cold water, saturated $\mathrm{NaHCO}_{3}$ solution, and saturated brines. After drying over anhydrous sodium sulfate, the solvent was removed under reduced pressure. A crude product was chromatographed on silica gel (elution: $V_{\text {petroleum ether }}: V_{\text {ethyl acetate }}=2: 1$ ) to give $285 \mathrm{mg}$ of compound $10(42.3 \%)$ as a white solid. IR (KBr) $v / \mathrm{cm}^{-1}: 1165,1434,1501$, 1589, 1733, 2930, 3429; ${ }^{1} \mathrm{H}$ NMR $\left(\mathrm{CDCl}_{3}, 600 \mathrm{MHz}\right): 0.655$ $\left(1 \mathrm{H}, \mathrm{s}, 18-\mathrm{CH}_{3}\right), 0.896\left(3 \mathrm{H}, \mathrm{d}, J=6.6,21-\mathrm{CH}_{3}\right), 0.946(3 \mathrm{H}$, $\left.\mathrm{s}, 19-\mathrm{CH}_{3}\right), 2.36-2.31(1 \mathrm{H}, \mathrm{m}, \mathrm{C} 23-\mathrm{H}), 2.55-2.52(1 \mathrm{H}, \mathrm{m}, \mathrm{C} 23-$ $\mathrm{H}), 2.894(1 \mathrm{H}$, br s, OH), $3.060(1 \mathrm{H}, \mathrm{dd}, J=15.6,13.2, \mathrm{C} 2-\beta \mathrm{H})$, $3.636\left(3 \mathrm{H}, \mathrm{s}, \mathrm{OCH}_{3}\right), 3.932(1 \mathrm{H}$, br s, C7-H), $6.309(1 \mathrm{H}, \mathrm{d}, J=$ $\left.4.2,-\mathrm{NH}_{2}\right), 7.281\left(1 \mathrm{H}, \mathrm{d}, J=4.2,-\mathrm{NH}_{2}\right), 9.135$ (1H, s, -NH-); ${ }^{13} \mathrm{C} \mathrm{NMR}\left(\mathrm{CDCl}_{3}, 150 \mathrm{MHz}\right): 11.9$ (18-C), 18.4 (19-C), 21.2 (21C), 22.4 (11-C), 23.8 (15-C), 28.2 (1-C), 30.2 (2-C), 31.0 (16-C), 31.1 (23-C), 31.2 (22-C), 33.2 (4-C), 33.7 (20-C), 35.5 (6-C), 35.9 (12-C), 37.4 (10-C), 39.6 (8-C), 39.7 (9-C), 42.3 (13-C), 42.8 (5-C), 50.3 (14-C), $51.6\left(-\mathrm{OCH}_{3}\right), 55.9$ (17-C), 68.7 (7-C), 158.7 (3-C), 174.9 (24-C), 178.4 (C=S); HREIMS: $m / z 478.3108$ $[\mathrm{M}+\mathrm{H}]^{+}$(calcd for $\mathrm{C}_{26} \mathrm{H}_{44} \mathrm{~N}_{3} \mathrm{O}_{3} \mathrm{~S}, 478.3103$ ).

2.3.3. Preparation of Platinum(II) Complexes. Solution of steroidal thiosemicarbazone $(0.1 \mathrm{mmol})$ in methanol $(8 \mathrm{~mL})$ and $0.1 \mathrm{mmol} \mathrm{K}_{2} \mathrm{PtCl}_{4}$ was added in the round bottom flask. The mixture was stirred for 5 hours at $70^{\circ} \mathrm{C}$. The reaction was terminated when large precipitant was emerged. The resulting suspension was filtered, washed with ethyl acetate and water, and dried in a desiccator over phosphorus pentoxide to give target products.

[PtL ${ }^{2} \mathrm{Cl}_{2}$ ] (Compound 3). Light yellow solid, Yield: 48.5\%; m.p. 297-299 ${ }^{\circ} \mathrm{C}$; IR (KBr) $v / \mathrm{cm}^{-1}$ : 3416, 2927, 1609, 1584, 1499, 1409, 1160, 878; ${ }^{1} \mathrm{H}$ NMR (300 MHz, DMSO) $\delta: 0.90(3 \mathrm{H}, \mathrm{s}$, $\left.18-\mathrm{CH}_{3}\right), 0.94\left(0.9 \mathrm{H}, \mathrm{s}, 18-\mathrm{CH}_{3}\right), 2.77-2.57(2 \mathrm{H}, \mathrm{m}, \mathrm{Cl}-\mathrm{H})$, $6.46(1 \mathrm{H}, \mathrm{d}, J=2.4, \mathrm{C} 4-\mathrm{H}), 6.52(1 \mathrm{H}, \mathrm{dd}, J=8.4,2.4, \mathrm{C} 2-\mathrm{H})$, $7.03(0.31 \mathrm{H}, \mathrm{d}, J=9.0, \mathrm{C} 1-\mathrm{H}), 7.07(0.69 \mathrm{H}, \mathrm{d}, J=8.4, \mathrm{C} 1-\mathrm{H})$, $8.38\left(1 \mathrm{H}, \mathrm{s},-\mathrm{NH}_{2}\right), 8.99\left(1 \mathrm{H}, \mathrm{s},-\mathrm{NH}_{2}\right), 10.77(1 \mathrm{H}, \mathrm{s},-\mathrm{NH}-)$; ${ }^{13} \mathrm{C}$ NMR (75 MHz, DMSO) $\delta: 12.5$ (18-C), 14.2 (18-C), 20.9 (11-C), 23.6 (15-C), 26.3 (16-C), 26.8 (7-C), 29.1 (6-C), 31.8 (16C), 35.2 (12-C), 35.4 (12-C), 37.5 (9-C), 40.1 (8-C), 43.1 (13C), 49.4 (13-C), 53.6 (14-C), 59.9 (14-C), 112.9 (2-C), 115.0 (4C), 126.0 (1-C), 130.1 (10-C), 137.1 (5-C), 155.1 (3-C), 170.5 (17C), 185.9 (C=S).; HREIMS: $m / z 607.0728[\mathrm{M}+\mathrm{H}]^{+}($calcd for $\left.\mathrm{C}_{19} \mathrm{H}_{26} \mathrm{Cl}_{2} \mathrm{~N}_{3} \mathrm{OPtS}, 607.0665\right)$.

[PtL ${ }^{5} \mathrm{Cl}_{2}$ ] (Compound 6). Yellow solid, Yield: 51.2\%; m.p. $287-288^{\circ} \mathrm{C}$; IR (KBr) $v / \mathrm{cm}^{-1}$ : 3329, 3129, 1763, 1626, 1556, $1489,1202,941,811 ;{ }^{1} \mathrm{H}$ NMR (600 MHz, DMSO) $\delta: 0.91(3 \mathrm{H}$, s, $\left.18-\mathrm{CH}_{3}\right), 2.23\left(3 \mathrm{H}, \mathrm{s}, \mathrm{COCH}_{3}\right), 2.63(1 \mathrm{H}, \mathrm{dd}, J=19.2,9.0$, C6-H), 2.84-2.81 (2H, m, C16-H), 6.80 (1H, d, J = 2.4, C4-H), $6.85(1 \mathrm{H}, \mathrm{dd}, J=8.4,2.4, \mathrm{C} 2-\mathrm{H}), 7.32(1 \mathrm{H}, \mathrm{d}, J=8.4, \mathrm{C} 1-\mathrm{H})$, $8.40\left(1 \mathrm{H}, \mathrm{s},-\mathrm{NH}_{2}\right), 8.99\left(1 \mathrm{H}, \mathrm{s},-\mathrm{NH}_{2}\right), 10.78(1 \mathrm{H}, \mathrm{s},-\mathrm{NH}-)$; HREIMS: $[\mathrm{M}+\mathrm{H}]^{+} 619.1162$ (calcd for $\mathrm{C}_{21} \mathrm{H}_{27} \mathrm{KN}_{3} \mathrm{O}_{2} \mathrm{PtS}$, 619.1109).

[PtL ${ }^{8} \mathrm{Cl}_{2}$ ] (Compound 9). Light yellow solid, Yield: 46.7\%, m.p. $270-272^{\circ} \mathrm{C}$; IR (KBr) $v / \mathrm{cm}^{-1}$ : 1706, 1624, 1437, 1329; ${ }^{1} \mathrm{H}$ $\mathrm{NMR}\left(\mathrm{CDCl}_{3}, 600 \mathrm{MHz}\right): 0.62\left(3 \mathrm{H}, \mathrm{s}, 18-\mathrm{CH}_{3}\right), 0.87(3 \mathrm{H}, \mathrm{d}$,
$\left.J=6.6,21-\mathrm{CH}_{3}\right), 1.17\left(1.8 \mathrm{H}, \mathrm{s}, 19-\mathrm{CH}_{3}, \mathrm{R}-\right), 1.20(1.2 \mathrm{H}, \mathrm{s}, 19-$ $\left.\mathrm{CH}_{3}, \mathrm{~S}-\right), 2.91(0.4 \mathrm{H}, \mathrm{dd}, J=12.6,4.8, \mathrm{C} 6-\beta \mathrm{H}, \mathrm{S}-), 2.96(0.6 \mathrm{H}$, $\mathrm{dd}, J=12.6,6.0, \mathrm{C} 6-\beta \mathrm{H}, \mathrm{R}-), 3.57\left(3 \mathrm{H}, \mathrm{s}, \mathrm{OCH}_{3}\right), 6.79(1 \mathrm{H}, \mathrm{s}$, $\left.-\mathrm{NH}_{2}\right), 6.84\left(1 \mathrm{H}, \mathrm{s},-\mathrm{NH}_{2}\right) ;{ }^{13} \mathrm{C} \mathrm{NMR}\left(\mathrm{CDCl}_{3}, 150 \mathrm{MHz}\right): 11.9$ (18-C), 18.3 (19-C), 21.6 (21), 21.9 (11-C), 22.0 (1-C), 24.4 (15C), 27.9 (16-C), 30.4 (2-C), 30.7 (23-C), 34.8 (22-C), 35.2 (4-C, S-), 35.5 (4-C, R-), 36.3 (20-C), 38.3 (12-C), 42.1 (10-C), 42.23 (6-C, S-), 42.29 (6-C, R-), 42.7 (13-C), 44.0 (5-C, S-), 44.6 (5C, R-), 46.3 (14-C), 48.4 (8-C, S-), 48.6 (8-C, R-), 49.1 (9-C), $51.3\left(\mathrm{OCH}_{3}\right), 54.3$ (17-C), 168.0 (3-C, $R$-), 168.1 (3-C, S-), 173.8 (24-C), 175.4 (C=S, S-), 176.0 (C=S, R-), 211.3 (7-C); HREIMS: $m / z 669.2430[\mathrm{M}]^{+}$(calcd for $\mathrm{C}_{26} \mathrm{H}_{40} \mathrm{~N}_{3} \mathrm{O}_{3} \mathrm{PtS}$, 669.2438).

[PtL ${ }^{10} \mathrm{Cl}_{2}$ ] (Compound 11). Light yellow solid, Yield: 51.1\%, m.p. 272-274 ${ }^{\circ} \mathrm{C}$; IR (KBr) $v / \mathrm{cm}^{-1}: 3434,2932,1733,1629,1437$, 1329; ${ }^{1} \mathrm{H}$ NMR (DMSO, $\left.600 \mathrm{MHz}\right)$ : $0.62\left(2.1 \mathrm{H}, \mathrm{s}, 18-\mathrm{CH}_{3}, \mathrm{R}-\right)$, $0.63\left(0.9 \mathrm{H}, \mathrm{s}, 18-\mathrm{CH}_{3}, S-\right), 0.88\left(2.1 \mathrm{H}, \mathrm{s}, 19-\mathrm{CH}_{3}, R-\right), 0.89(3 \mathrm{H}$, $\left.\mathrm{d}, J=6.6,21-\mathrm{CH}_{3}\right), 0.93\left(0.9 \mathrm{H}, \mathrm{s}, 19-\mathrm{CH}_{3}, S-\right), 2.23-2.16(1 \mathrm{H}$, $\mathrm{m}, \mathrm{C} 23-\mathrm{H}), 2.35-2.30(1 \mathrm{H}, \mathrm{m}, \mathrm{C} 23-\mathrm{H}), 3.57\left(3 \mathrm{H}, \mathrm{s}, \mathrm{OCH}_{3}\right)$, 3.69-3.61 (1H, m, C7-H), $6.62\left(0.3 \mathrm{H}, \mathrm{s},-\mathrm{NH}_{2}, \mathrm{~S}-\right), 6.50(0.3 \mathrm{H}$, $\left.\mathrm{s},-\mathrm{NH}_{2}, S-\right), 6.70\left(0.7 \mathrm{H}, \mathrm{s},-\mathrm{NH}_{2}, R-\right), 6.76\left(0.7 \mathrm{H}, \mathrm{s},-\mathrm{NH}_{2}, R-\right)$; ${ }^{13} \mathrm{C}$ NMR (DMSO, $\left.150 \mathrm{MHz}\right): 11.7$ (18-C), 18.2 (19-C), 20.6 (21C), 21.9 (11-C), 23.2 (15-C), 27.8 (1-C), 30.4 (2-C), 30.7 (16-C), 32.1 (23-C), 32.9 (22-C), 33.7 (4-C, S-), 33.9 (4-C, R-), 34.4 (20C 5-C), 34.9 (6-C), 35.4 (12-C), 37.8 (10-C), 40.5 (8-C), 42.0 (9-C), 42.1 (13-C), 45.2 (5-C), 49.9 (14-C), $51.3\left(\mathrm{OCH}_{3}\right), 55.5$ (17-C), 66.2 (7-C), 167.4 (3-C, S-), 167.8 (3-C, R-), 173.9 (24C), 177.8 (C=S, $R$-), 178.4 (C=S, S-); HREIMS: $m / z 695.2554$ $[\mathrm{M}+\mathrm{Na}]^{+}$(calcd for $\mathrm{C}_{26} \mathrm{H}_{43} \mathrm{~N}_{3} \mathrm{NaO}_{3} \mathrm{PtS}, 695.2570$ ).

[PtL ${ }^{13} \mathrm{Cl}_{2}$ ] (Compound 14). Light yellow solid, Yield: 41.0\%, m.p. $270-272^{\circ} \mathrm{C}$; IR (KBr) $v / \mathrm{cm}^{-1}: 3426,1736,1706,1616,1447$; ${ }^{1} \mathrm{H}$ NMR (DMSO, $\left.600 \mathrm{MHz}\right): 0.75\left(1.4 \mathrm{H}, \mathrm{s}, 18-\mathrm{CH}_{3}, \mathrm{~S}-\right), 0.76$ $\left(1.6 \mathrm{H}, \mathrm{s}, 18-\mathrm{CH}_{3}, \mathrm{R}-\right), 0.97\left(3 \mathrm{H}, \mathrm{s}, 19-\mathrm{CH}_{3}\right), 1.00(3 \mathrm{H}, \mathrm{d}, J=$ 7.2, 21- $\left.\mathrm{CH}_{3}\right), 2.78-2.68(1 \mathrm{H}, \mathrm{m}, \mathrm{C} 20-\mathrm{H}), 3.19(0.46 \mathrm{H}, \mathrm{d}, J=$ 13.2, C2-H, S-), 3.46 (0.54H, d, J = 15.0, C4-H, R-), 3.57 (3H, $\left.\mathrm{s}, \mathrm{OCH}_{3}\right), 6.75\left(1 \mathrm{H}\right.$, br s, $\left.-\mathrm{NH}_{2}\right), 6.79\left(1 \mathrm{H}\right.$, br s, $\left.-\mathrm{NH}_{2}\right) ;{ }^{13} \mathrm{C}$ NMR (DMSO, $150 \mathrm{MHz})$ : 11.3 (18-C), 18.6 (19-C), 23.9 (21-C), 25.0 (15-C), 26.3 (16-C), 27.1 (1-C), 30.3 (2-C), 30.7 (6-C), 35.1 (7-C), 35.4 (22-C), 35.7 (23-C), 36.5 (4-C, S-), 36.7 (4-C, R-), 37.3 (20-C), 38.0 (11-C), 42.8 (8-C), 43.5 (10-C), 45.4 (13-C), 46.3 (5-C), $51.3\left(\mathrm{OCH}_{3}\right), 56.8$ (9-C), 57.3 (17-C), 57.4 (14-C), 167.7 (3-C, S-), 168.1 (3-C, R-), 173.7 (24-C), 177.0 (C=S, S-), 177.6 (C=S, R-), 211.5 (12-C, S-), 213.4 (12-C, R-); HREIMS: $m / z 671.2555[\mathrm{M}+\mathrm{H}]^{+}$(calcd for $\mathrm{C}_{26} \mathrm{H}_{42} \mathrm{~N}_{3} \mathrm{O}_{3} \mathrm{PtS}, 671.2595$ ).

2.4. Cytotoxicity Assay In Vitro. The antiproliferative activity of all steroidal thiosemicarbazones and their $\mathrm{Pt}(\mathrm{II})$ metal complexes and cisplatin on Bel 7404 (human liver carcinoma), HeLa (human cervical carcinoma), and HEK293T (normal kidney epithelial cells) cell lines was determined by using the MTT method. The detailed procedure has been reported in our previous work [24].

\section{Results and Discussion}

3.1. Synthesis and Characterization. The synthetic route and the structures of complexes $\mathbf{3}$ and $\mathbf{6}$ were outlined in Scheme 1. Steroidal thiosemicarbazones $\mathbf{2}$ and $\mathbf{5}$ were 


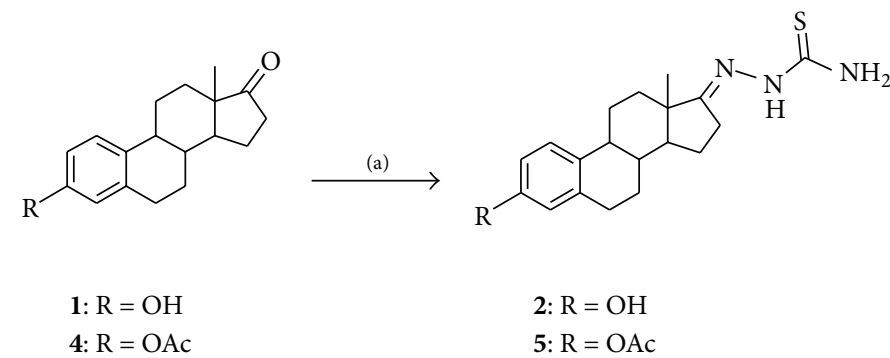<smiles>[R]O[R4]([R])=O</smiles>

(S)-configuration

Reagents and conditions:

(a) thiosemicarbazide, acetic acid, and ethanol

(b) $\mathrm{K}_{2} \mathrm{PtCl}_{4}, \mathrm{CH}_{3} \mathrm{OH} / \mathrm{CHCl}_{3}=1 / 1$

SCHEME 1: Synthesis of estrone-17-thiosemicarbazone platinum(II).

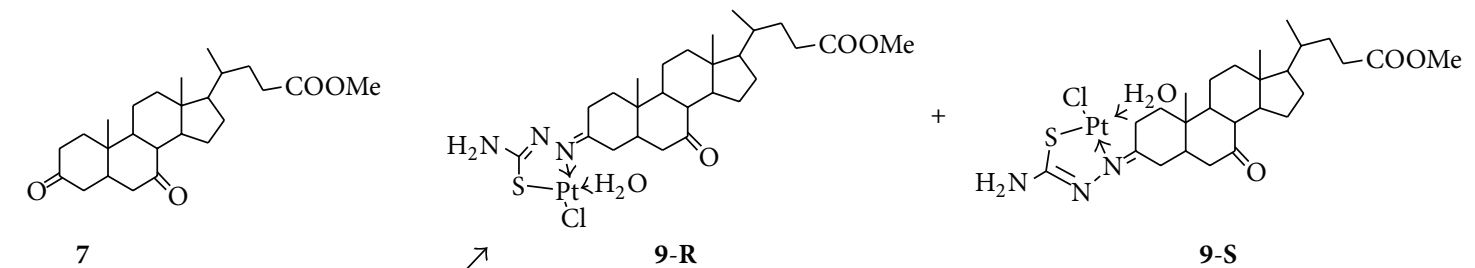

7

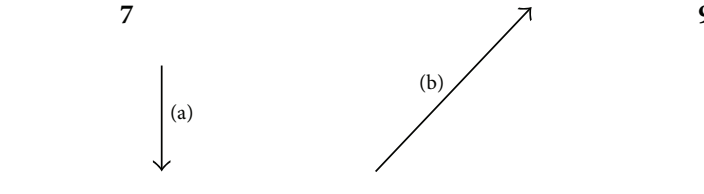

9-R

9-S

(c)

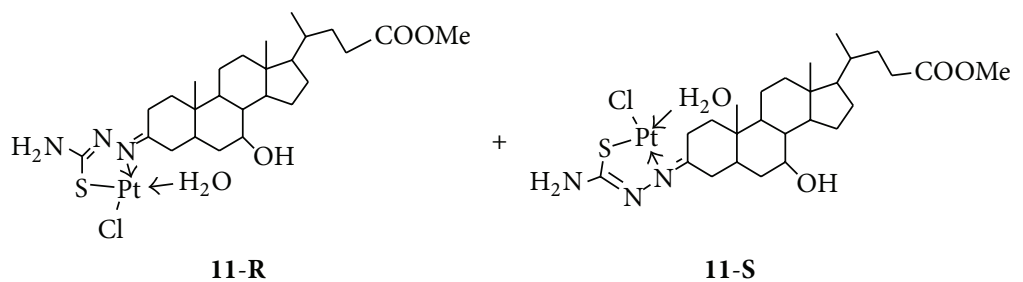

Reagents and conditions:

(a) thiosemicarbazide, acetic acid, and ethanol

(b) $\mathrm{K}_{2} \mathrm{PtCl}_{4}, \mathrm{CH}_{3} \mathrm{OH} / \mathrm{CHCl}_{3}=1 / 1$

(c) $\mathrm{NaBH}_{4}, \mathrm{CH}_{3} \mathrm{OH}$

(d) $\mathrm{K}_{2} \mathrm{PtCl}_{4}, \mathrm{CH}_{3} \mathrm{OH} / \mathrm{CHCl}_{3}=1 / 1$

Scheme 2: Synthesis of methyl chenodeoxycholate complexes 9 and $\mathbf{1 0 .}$

obtained as a $(E)$-configuration by reacting estrone with thiosemicarbazide, and the reaction of compounds $\mathbf{2}$ and $\mathbf{5}$ with $\mathrm{K}_{2} \mathrm{PtCl}_{4}$ gave steroidal platinum (Pt(II)) complexes 3 and $\mathbf{6}$ as $(S)$-configuration, respectively. The structures of $\mathbf{3}$ and $\mathbf{6}$ were confirmed by analysis of UV, IR, NMR, and HRMS.
In order to investigate the effect of position of pharmacophore on the antiproliferative activity of complexes, we prepared complexes $\mathbf{9}$ and $\mathbf{1 1}$ using chenodeoxycholic acid as a starting material (Scheme 2). Starting from compound 7, compound 8 with 3 -thiosemicarbazone group was yielded as a mixture of $(3 E)$ - and (3Z)-isomer (ratio: $0.6: 0.4,{ }^{1} \mathrm{H}$ 
<smiles>CC(=O)CCC(C)C1CCC2C3CCC4CC(=O)CCC4(C)C3CC(=O)C12C(C)CCC(C)=O</smiles>

12<smiles></smiles>

14-R<smiles></smiles>

14-S

Reagents and conditions:

(a) thiosemicarbazide, acetic acid, and ethanol

(b) $\mathrm{K}_{2} \mathrm{PtCl}_{4}, \mathrm{CH}_{3} \mathrm{OH} / \mathrm{CHCl}_{3}=1 / 1$

Scheme 3: Synthesis of methyl 7-deoxycholate complex 14.

NMR data) by controlling an appropriate molar ratio of 7 and thiosemicarbazide because 3-carbonyl group was more active than 7-carbonyl, and thiosemicarbazide was selectively reacted with 3-carbonyl. The reaction of compound 8 with $\mathrm{K}_{2} \mathrm{PtCl}_{4}$ afforded further complex 9 as a mixture of $(R)$ - and $(S)$-configuration isomer (9-R: 9-S = 3:2, ${ }^{1} \mathrm{H}$ NMR data).

Next, the 7-carbonyl of $\mathbf{8}$ was converted to 7-hydroxyl of compound $\mathbf{1 0}$ by the reduction of $\mathrm{NaBH}_{4}$, but the 3thiosemicarbazone group was still kept in compound 10. The reaction of 10 with $\mathrm{K}_{2} \mathrm{PtCl}_{4}$ gave complex 11. Complex 11 was a mixture of $(R)$ - and $(S)$-configuration isomer also (9-R: 9-S = 7:3, ${ }^{1} \mathrm{H}$ NMR data).

The structures of complexes $\mathbf{9}$ and $\mathbf{1 1}$ had been determined by analysis of IR, UV, NMR, and HRMS.

Using 7-deoxycholic acid as a starting material, another steroidal thiosemicarbazone platinum ( $\mathrm{Pt}(\mathrm{II})$ ) complex 14 was synthesized (Scheme 3). Similarly, complex 14 was a mixture of $(R)$ - and $(S)$-configuration isomer (14-R: $\mathbf{1 4}-\mathbf{S}=$ 0.54:0.46, ${ }^{1} \mathrm{H}$ NMR data) and their structures had been confirmed by analysis of IR, UV, NMR, and HRMS.

3.2. Cytotoxic Activity In Vitro. The antiproliferative activities of all steroidal thiosemicarbazones and their $\mathrm{Pt}(\mathrm{II})$ metal complexes were determined in vitro on Bel 7404, HeLa, and HEK293T. The MTT method was used to assay the antiproliferative activity and cisplatin was used as a positive control. The results are summarized as $\mathrm{IC}_{50}$ values in $\mu \mathrm{M}$ in Table 1 .

As shown in Table 1, steroidal platinum (Pt(II)) complexes 9, 11, and 14 are almost inactive against Bel 7404 and HeLa cells. However, complexes $\mathbf{3}$ and $\mathbf{6}$ exhibited an obvious cytotoxicity to HeLa and Bel 7404 cells. In particular, complex 6 showed an excellent antiproliferative activity against HeLa cells with the $\mathrm{IC}_{50}$ values of $9.2 \mu \mathrm{M}$ and had a better cytotoxicity compared with its precursor.
Table 1: Cytotoxicity ${ }^{\mathrm{a}}$ of steroidal thiosemicarbazones and their Ptcomplexes in vitro $\left(\mathrm{IC}_{50}: \mu \mathrm{M}\right)^{\mathrm{b}}$.

\begin{tabular}{lccc}
\hline Compounds & Bel-7404 & HeLa & HEK293T \\
\hline $\mathbf{2}$ & 19 & 34 & ND \\
$\mathbf{3}$ & 39 & 23 & $>200$ \\
$\mathbf{5}$ & $>200$ & 42 & $>200$ \\
$\mathbf{6}$ & 86 & 9.2 & $>200$ \\
$\mathbf{8}$ & $>200$ & 96 & $>200$ \\
$\mathbf{9}$ & $>200$ & 156 & $>200$ \\
$\mathbf{1 0}$ & $>200$ & 142 & $>200$ \\
$\mathbf{1 1}$ & $>200$ & $>200$ & $>200$ \\
$\mathbf{1 3}$ & $>200$ & $>200$ & $>200$ \\
$\mathbf{1 4}$ & 103 & 102 & $>200$ \\
Cisplatin & 23.2 & 10.1 & 10.3 \\
\hline
\end{tabular}

${ }^{a}$ Cytotoxicity as $\mathrm{IC}_{50}$ for each cell line is the concentration of compound which reduced by $50 \%$ the optical density of treated cells with respect to untreated cells using the MTT assay.

${ }^{\mathrm{b}}$ Data represent the mean values of three independent determinations.

Comparing the antiproliferative activity of estrone-17thiosemicarbazone platinum(II) with that of methyl chenodeoxycholate-thiosemicarbazone platinum(II) and methyl 7deoxycholate-thiosemicarbazone platinum(II), we can see that estrone-17-thiosemicarbazone platinum(II) shows a better inhibiting activity than methyl chenodeoxycholate-thiosemicarbazone platinum(II) and methyl 7-deoxycholatethiosemicarbazone platinum(II). A reason is that estrone17-thiosemicarbazone platinum(II) with the structure of steroidal nucleus of estrone may be connected with the metabolism of estrogen. 
TABLE 2: SI values of steroidal thiosemicarbazone Pt-complexes.

\begin{tabular}{lcccccc}
\hline Compounds & $\mathbf{3}$ & $\mathbf{6}$ & $\mathbf{9}$ & $\mathbf{1 1}$ & $\mathbf{1 4}$ & Cisplatin \\
\hline $\mathrm{SI}_{\text {Bel 7404 }}$ & 5.1 & 2.3 & - & - & 1.9 & - \\
$\mathrm{SI}_{\text {HeLa }}$ & 8.7 & 21.7 & 1.3 & - & 2.0 & 1.0 \\
\hline
\end{tabular}

Here, the positive control cisplatin and complex 6 displayed similar cytotoxicity against HeLa cells but cisplatin had obvious cytotoxicity to normal kidney epithelial cells HEK293T and complex $\mathbf{6}$ was almost inactive.

The Selectivity Index (SI) was defined as the ratio of the cytotoxicity of a compound with respect to normal cells $\left(\mathrm{IC}_{50}\right.$ HEK293T) versus cancer cells and used to determine the criterion of effectiveness of the compounds. The SI values of the complexes are listed in Table 2.

One important criterion for a therapeutic drug for cancer is to have minimal or no side effects to normal body cells of patients undergoing chemotherapy. Considering that a higher SI corresponds to greater overall anticancer activity, we can affirm that complex $\mathbf{6}$ is an excellent selective inhibitor against HeLa cells, which deserve further study (SI value: complex 6 21.7, cisplatin 1.0).

\section{Conclusion}

In conclusion, we had prepared some steroidal thiosemicarbazone platinum(II) complexes and assayed their antiproliferative activities. The results showed that estrone-17thiosemicarbazone platinum(II) displayed a better inhibiting activity than methyl chenodeoxycholate-thiosemicarbazone platinum(II) and methyl 7-deoxycholate- thiosemicarbazone platinum(II). Among them, complex $\mathbf{6}$ based on the structure of estrone was found to be a valuable selective inhibitor against HeLa cells possessing the $\mathrm{IC}_{50}$ values of $9.2 \mu \mathrm{M}$ and SI value of 21.7. The result may be useful for the design of novel chemotherapeutic drugs.

\section{Conflict of Interests}

The authors declare that there is no conflict of interests regarding the publication of this paper.

\section{Acknowledgments}

The authors acknowledge the financial support of the Natural Science Fund of Education Department of Guangxi Province (no. 201202ZD059) and Scientific Developing Fund of Nanning (no. 20145203).

\section{References}

[1] B. Rosenberg, L. Van Camp, and T. Krigas, "Inhibition of cell division in Escherichia coli by electrolysis products from a platinum electrode," Nature, vol. 205, no. 4972, pp. 698-699, 1965.

[2] B. Rosenberg, L. VanCamp, J. E. Trosko, and V. H. Mansour, "Platinum compounds: a new class of potent antitumour agents," Nature, vol. 222, no. 5191, pp. 385-386, 1969.
[3] P. J. Loehrer and L. H. Einhorn, "Drugs five years later. Cisplatin," Annals of Internal Medicine, vol. 100, no. 5, pp. 704713, 1984.

[4] Z. Guo and P. J. Sadler, "Metals in Medicine," Angewandte Chemie International Edition, vol. 38, no. 11, pp. 1512-1531, 1999.

[5] K. Barabas, R. Milner, D. Lurie, and C. Adin, "Cisplatin: a review of toxicities and therapeutic applications," Veterinary and Comparative Oncology, vol. 6, no. 1, pp. 1-18, 2008.

[6] R. B. R. Porter, W. A. Gallimore, and P. B. Reese, "Steroid transformations with Exophiala jeanselmei var. lecanii-corni and Ceratocystis paradoxa," Steroids, vol. 64, no. 11, pp. 770-779, 1999.

[7] M. R. Wilson, W. A. Gallimore, and P. B. Reese, "Steroid transformations with Fusarium oxysporum var. cubense and Colletotrichum musae," Steroids, vol. 64, no. 12, pp. 834-843, 1999.

[8] G. Hayem, O. Domarle, M. Fay, M. Thuong-Guyot, J.-J. Pocidalo, and C. Carbon, "Lack of correlation between hydrogen peroxide production and nitric oxide production by cultured rabbit articular chondrocytes treated with fluoroquinolone antimicrobial agents," Toxicology in Vitro, vol. 10, no. 5, pp. 551$555,1996$.

[9] F. Rouillon, G. Berdeaux, J. C. Bisserbe et al., "Prevention of recurrent depressive episodes with milnacipran: consequences on quality of life," Journal of Affective Disorders, vol. 58, no. 3, pp. 171-180, 2000.

[10] D. Rosi, G. Peruzzotti, E. W. Dennis, D. A. Berberian, H. Freele, and S. Archer, "A new, active metabolite of 'Miracil D"', Nature, vol. 208, no. 5014, pp. 1005-1006, 1965.

[11] C. G. Wermuth, "Aminopyridazines - an alternative route to potent muscarinic agonists with no cholinergic syndrome," Farmaco, vol. 48, no. 2, pp. 253-274, 1993.

[12] G. A. G. Santos, A. P. Murray, C. A. Pujol, E. B. Damonte, and M. S. Maier, "Synthesis and antiviral activity of sulfated and acetylated derivatives of $2 \beta, 3 \alpha$-dihydroxy- $5 \alpha$-cholestane," Steroids, vol. 68, no. 2, pp. 125-132, 2003.

[13] Y. Ueno, Y. Okamoto, R. Yamauchi, and K. Kato, "An antitumor activity of the alkali-soluble polysaccharide (and its derivatives) obtained from the sclerotia of Grifora umbellata (Fr.) pilát," Carbohydrate Research, vol. 101, no. 1, pp. 160-167, 1982.

[14] Z. H. Chohan, "Biologically active transition metal chelates of $\mathrm{Ni}(\mathrm{II}), \mathrm{Cu}(\mathrm{II})$ and $\mathrm{Zn}(\mathrm{II})$ with 2-aminothiazole-derived schiff-bases: their synthesis, characterization and the role of anions $\left(\mathrm{NO}_{3}, \mathrm{SO}_{4}^{2-}, \mathrm{C}_{2} \mathrm{O}_{4}^{2-}\right.$ and $\left.\mathrm{CH}_{3} \mathrm{CO}_{2-}\right)$ on their antibacterial properties," Metal-Based Drugs, vol. 6, no. 3, pp. 187-192, 1999.

[15] S. Adsule, S. Banerjee, F. Ahmed, S. Padhye, and F. H. Sarkar, "Hybrid anticancer agents: isothiocyanate-progesterone conjugates as chemotherapeutic agents and insights into their cytotoxicities," Bioorganic and Medicinal Chemistry Letters, vol. 20, no. 3, pp. 1247-1251, 2010.

[16] R. Gust, W. Beck, G. Jaouen, and H. Schönenberger, "Optimization of cisplatin for the treatment of hormone dependent tumoral diseases. Part 1. Use of steroidal ligands," Coordination Chemistry Reviews, vol. 253, no. 21-22, pp. 2742-2759, 2009.

[17] Z. H. Siddik, "Cisplatin: mode of cytotoxic action and molecular basis of resistance," Oncogene, vol. 22, no. 47, pp. 7265-7279, 2003.

[18] W. Steven and P. A. Johnson, "Relationship between platinumDNA adduct formation and removal and cisplatin cytotoxicity in cisplatin-sensitive and -resistant human ovarian cancer cells," Cancer Research, vol. 54, pp. 5911-5916, 1994. 
[19] A. G. Quiroga and C. N. Ranninger, "Contribution to the SAR field of metallated and coordination complexes: studies of the palladium and platinum derivatives with selected thiosemicarbazones as antitumoral drugs," Coordination Chemistry Reviews, vol. 248, no. 1-2, pp. 119-133, 2004.

[20] S. A. Khan and M. Yusuf, "Synthesis, spectral studies and in vitro antibacterial activity of steroidal thiosemicarbazone and their palladium (Pd (II)) complexes," European Journal of Medicinal Chemistry, vol. 44, no. 5, pp. 2270-2274, 2009.

[21] A. Murugkar, B. Unnikrishnan, S. Padhye et al., "Hormone anchored metal complexes. 1. Synthesis, structure, spectroscopy and in vitro antitumor activity of testosterone acetate thiosemicarbazone and its metal complexes," Metal-Based Drugs, vol. 6, no. 3, pp. 177-182, 1999.

[22] Y. M. Huang, Q. C. Yao, J. G. Cui et al., "Syntheses of lactam derivatives of chenodeoxycholic acid and in vitro antiproliferative activity," Chemical Research in Chinese Universities, vol. 30, no. 4, pp. 605-613, 2014.

[23] Y. M. Huang, S. J. Chen, J. G. Cui et al., "Synthesis and cytotoxicity of A-homo-lactam derivatives of cholic acid and 7deoxycholic acid," Steroids, vol. 76, no. 7, pp. 690-694, 2011.

[24] C. Gan, J. Cui, S. Su et al., "Synthesis and antiproliferative activity of some steroidal thiosemicarbazones, semicarbazones and hydrozones," Steroids, vol. 87, pp. 99-107, 2014. 

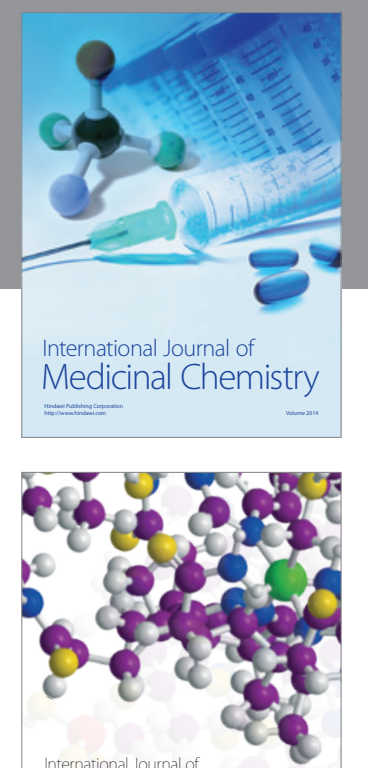

\section{Carbohydrate} Chemistry

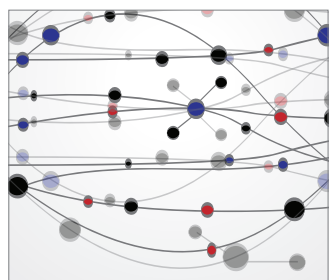

The Scientific World Journal
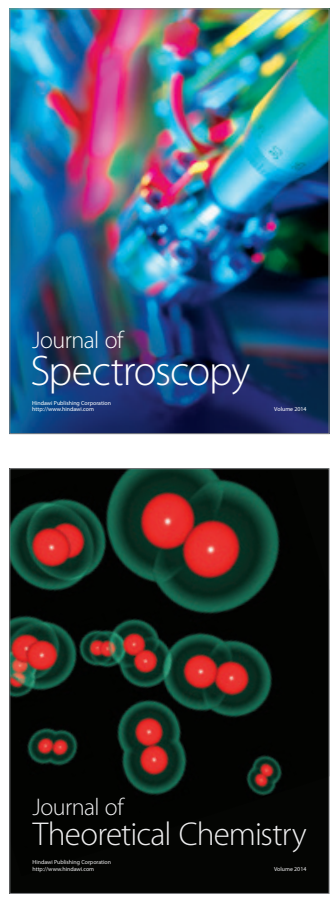
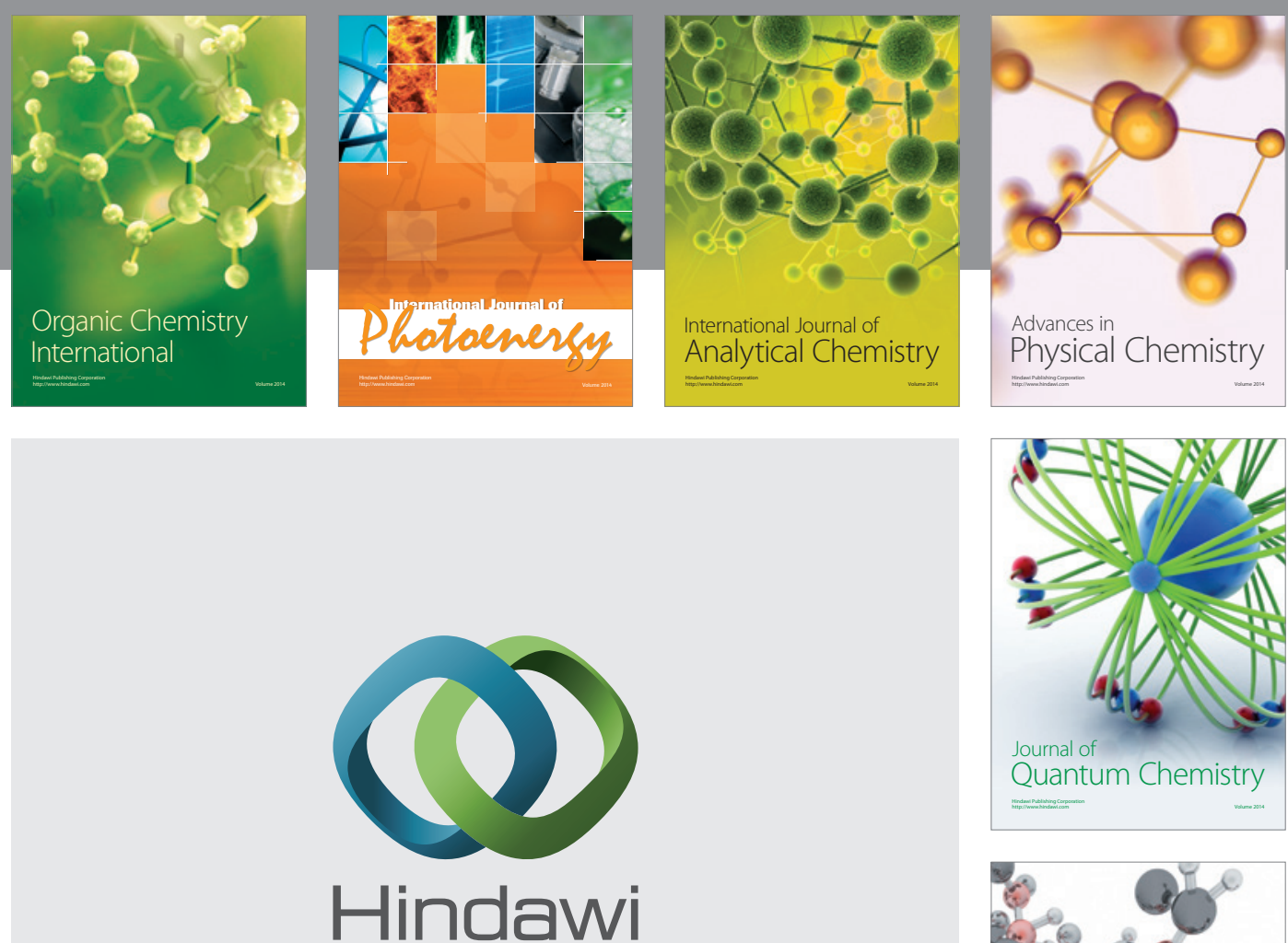

Submit your manuscripts at

http://www.hindawi.com

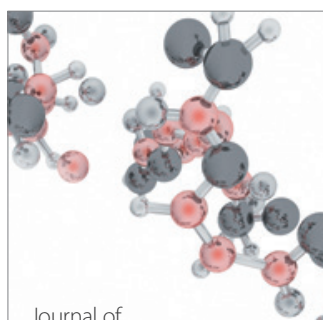

Analytical Methods

in Chemistry

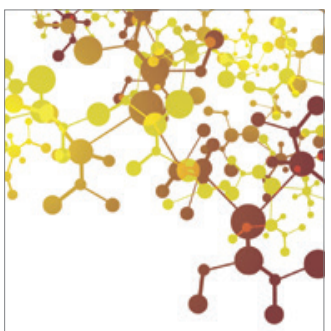

Journal of

Applied Chemistry

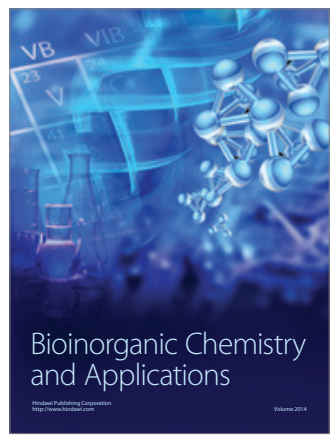

Inorganic Chemistry
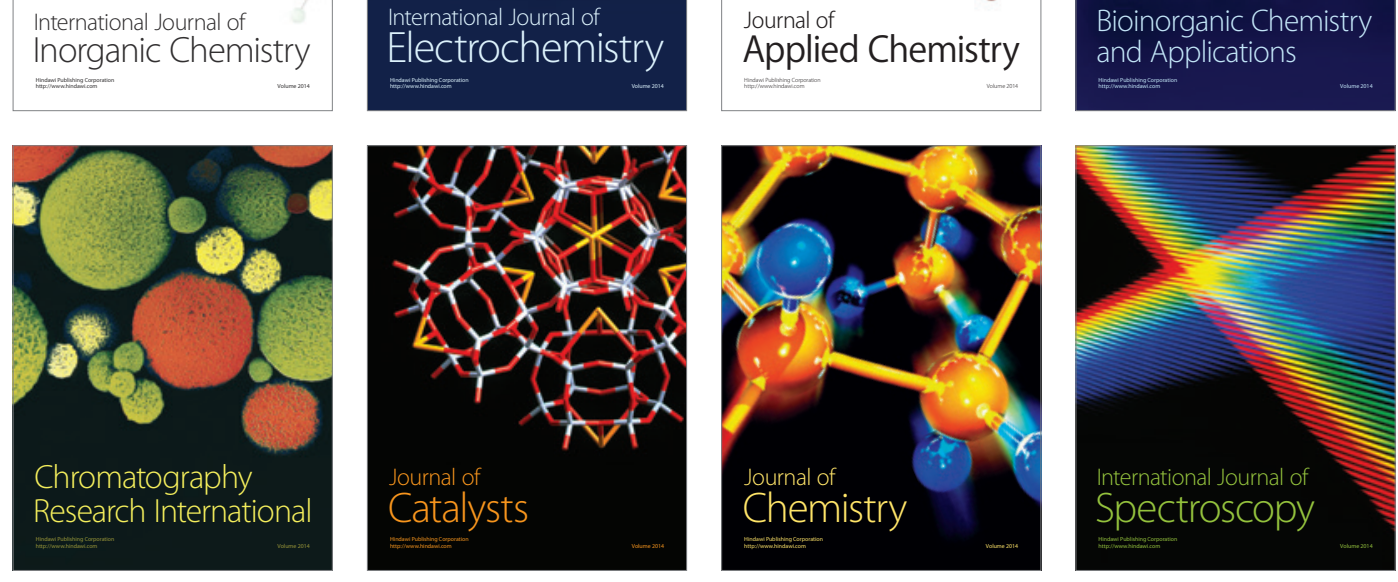